\title{
Establishment of Yemeni Dental Arch Form
}

\author{
Dr Nabil M. Al-Zubair \\ Professor, Department Orthodontics, Faculty of Dentistry, Sana'a University
}

Correspondence: dr.nabilzubair7@gmail.com

\section{ABSTRACT}

Objective: To assess the dental arch forms of Yemeni adult sample.

Materials \& Method: The Eucledian clustering method of analysis was utilized for the determination of dental arch form. A total of 398 study models were constructed and evaluated to do measurements for both arches using a modified sliding caliper gauge. Six dental cast measurements divided into three sagittal and three transverse measurements were utilized to represent the dental arch width and length measurements.

Result: Narrow form is the most prevalent arch form (30.9\%) followed by wide form (23.9\%), their prominence appear more in females and the least prevalent arch form was the mid form (9.3\%), while flat and pointed forms were in between $18.3 \%$ and $17.6 \%$ respectively.

Conclusion: Five arch forms: narrow, wide, mid, pointed and flat were distinguished as unique forms for the dental arches, with the predominance of the narrow arch form were found among Yemeni adults.

Key words: arch form, dental cast analysis, Yemeni dental Arch

\section{INTRODUCTION}

The search for the elusive "ideal" arch form to suit every individual has been the focus of several reports. However, dental arch form and shape varies in individuals based on anatomic dimensions of the craniofacial skeleton. ${ }^{1,2}$ Qualitative description of the dental arch use terms like elliptic, parabolic or U-shaped; whereas mathematical methods include several curve-fitting algorithms like parabola, semi ellipse, catenary's curve, conic section, cubic plane curve and second to eighth order polynomials. ${ }^{3,4}$ Despite the huge efforts and research works done to determine the ideal arch form, there is little agreement among investigators about the natural shape of the dental arches. ${ }^{5-7}$

Hawley; ${ }^{8}$ based on the earlier work of Bonwill ${ }^{9}$ described the ideal arch as being constructed upon an equilateral triangle with slight modifications. The six anterior teeth were thought to be arranged on the arc of a circle whose radius was determined by the combined width of incisors and canines, with the premolars and first permanent molars arranged in a straight line and the second and third molars turning towards the midline. Catenary arch design depends on inter-molar distance measured at central fossae, it could be described as a central core around which the teeth arrange themselves. ${ }^{10}$ Brader $^{3}$ proposed that the dental arch is best considered as a closed curve with the properties of a trifocal ellipse with the teeth occupying only the narrower end of the total curve.

Raberin et al " examined 278 dental casts of untreated French adults with normal occlusion. Six measurements of mandibular dental arch were performed, and five independent ratios were determined. They developed an arch form guide and defined five mandibular dental arch forms (narrow, wide, mid, pointed and flat).

Thus it is worthwhile to mention that determination of the dental arch form relies in the majority of the research works upon dental arch width and length dimensions. ${ }^{12}$

\section{MATERIALS AND METHOD}

The sample consisted of 199 adults aged between 18 to 26 years, among whom 97 were males and 102 females, selected from the clinical examination of 1703 Yemeni adults.

Following criteria were used for sample selection: full complement of permanent dentition (excluding third molars); Class I molar and canine relation; Class I skeletal relationship (decided visually by using two-finger technique); free of local factors that disturb the integrity of dental arches (e.g. congenital missing teeth, retained deciduous teeth, supernumerary teeth); normal overjet and overbite relation; no heavy restorations that may affect the dental arch size and form; no previous orthodontic or facial surgical treatment; well-aligned arches with less than $2 \mathrm{~mm}$ of spacing or crowding in either arch.

Measurements were taken from 398 maxillary and mandibular dental casts, which were made of dental stone with plaster of Paris base. The base was trimmed and numbered corresponding to the number given to the subjects. Dental arch dimensions were measured using the modified sliding calipers gauge of $0.02 \mathrm{~mm}$ accuracy. 
A: arch width

a: inter-canine distance

b: inter-first molar distance

c: inter-second molar distance

\section{B: arch length}

d: anterior arch length

e: molar-vertical distance

f: total arch length
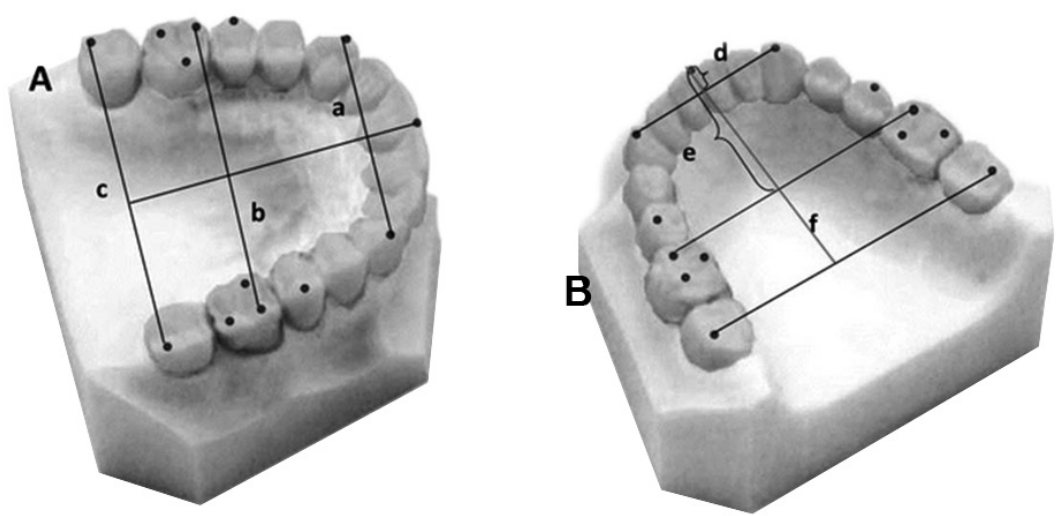

Figure 1: Dental Arch Dimensions

\section{Dental Arch Widths and Arch Lengths}

Dental cast measurements were divided into three sagittal and three transverse measurements; which were used to represent the dental arch width and length measurements (Figure 1).

\section{Dental Arch Forms}

Determination of dental arch form was done using the method used by Raberin et al:"1 which depends on the Eucledian clustering method of analysis. Six dental cast measurements were divided into three sagittal measurements and three transverse measurements; which were utilized to calculate five independent ratios. They are: anterior arch length/intercanine distance, molar-vertical distance/inter-first molar distance, total arch length/inter-second molar distance, intercanine distance/inter-first molar distance and molar-vertical distance/total arch length. Eucledian distance between the five ratios of each subject with the different centroid of clusters was calculated, each subject was allocated according to the nearest centriod, and as a result five arch forms were developed.

All statistical analyses were performed using SPSS 13.0 program. Descriptive statistics were performed for the calculation of the mean, standard deviation and coefficient of variance of the dental arch dimensions. Eucledian clustering analysis was used to identify the dental arch forms. Statistical significance was pre-determined at $95 \%$ level $(\mathrm{P}<0.05)$.

\section{RESULT}

The mean, standard deviation and range of the maxillary and mandibular dimensions are shown in Table 1. It can be noticed that the inter-second molar distances have the widest range. The range of reading is expressed by the coefficient of variation (CV) values. They reflect different arch size and forms. The inter-canine distance and anterior arch length showed the highest values of $\mathrm{CV}$ in both maxillary and mandibular dental arches, while the other related dimensions were close to each other (Table 1).

Table 1: Descriptive statistics for dental arch dimensions of the total sample (in $\mathrm{mm}$ )

\begin{tabular}{|l|c|c|c|c|c|c|c|}
\hline & Mean & SD & Min & Max & Range & CV \\
\hline \multicolumn{3}{|c|}{ Maxillary arch dimensions } \\
\hline Inter-canine distance & 34.2 & 2.04 & 30.0 & 39.0 & 9.0 & 5.40 \\
\hline Inter-first molar distance & 51.2 & 2.7 & 44.0 & 57.3 & 13.3 & 4.99 \\
\hline Inter-second molar distance & 56.9 & 3.3 & 49.4 & 64.7 & 15.3 & 5.18 \\
\hline Anterior arch length & 8.7 & 1.2 & 5.0 & 11.4 & 6.40 & 12.19 \\
\hline Molar-vertical distance & 30.0 & 2.1 & 24.9 & 34.9 & 10.0 & 6.79 \\
\hline Total arch length & 42.6 & 2.4 & 38.4 & 47.6 & 9.2 & 4.99 \\
\hline & Mandibular arch dimensions & & & & \\
\hline Inter-canine distance & 25.32 & 1.60 & 21.70 & 29.30 & 7.60 & 5.93 \\
\hline Inter-first molar distance & 44.02 & 2.60 & 37.50 & 49.60 & 12.10 & 5.06 \\
\hline Inter-second molar distance & 51.83 & 3.24 & 44.50 & 59.20 & 14.70 & 5.57 \\
\hline Anterior arch length & 5.09 & 1.09 & 1.70 & 7.56 & 5.86 & 13.57 \\
\hline Molar-vertical distance & 24.93 & 2.02 & 19.70 & 31.30 & 11.60 & 7.70 \\
\hline Total arch length & 38.36 & 2.74 & 33.00 & 45.10 & 12.10 & 5.79 \\
\hline
\end{tabular}


The correlation coefficient was carried out between arch widths and lengths, some of them showed high significant, positive and direct relations, while others showed moderate, weak or negative relationships. The correlations were clearly prominent and stronger between inter-canine distance and anterior arch length with other arch dimensions especially in female group as shown in Table 2.

Table 2: Correlation Coefficient between dental arch dimensions

\begin{tabular}{|l|c|c|c|c|c|c|c|}
\hline & \multicolumn{2}{|c|}{ Anterior arch length } & Molar-vertical distance & \multicolumn{2}{c|}{ Total arch length } \\
\cline { 2 - 7 } & \multicolumn{3}{|c|}{ Maxillary arch dimensions } & F & M & F & M \\
\hline Inter-canine distance & $0.47^{*}$ & 0.13 & $0.35^{*}$ & -0.11 & $0.30^{*}$ & -0.07 \\
\hline Inter-first molar distance & $0.56^{*}$ & 0.20 & $0.32^{*}$ & 0.01 & $0.24^{*}$ & 0.10 \\
\hline Inter-second molar distance & 0.18 & -0.12 & $0.24^{*}$ & 0.21 & 0.10 & 0.05 \\
\hline & Mandibular & arch dimensions & & & \\
\hline Inter-canine distance & $0.38^{*}$ & 0.17 & $0.44^{*}$ & $0.34^{*}$ & $0.50^{*}$ & $0.33^{*}$ \\
\hline Inter-first molar distance & -0.02 & 0.03 & -0.02 & 0.14 & 0.05 & 0.20 \\
\hline Inter-second molar distance & -0.09 & -0.05 & -0.09 & 0.15 & -0.07 & 0.10 \\
\hline
\end{tabular}

* Values more than 0.21 were significant at $p<0.01$

Table 3 shows the average of the five ratios used in the determination of dental arch forms. The percentage of relative deviation between the mean value of a given ratio for arch form and the mean value of the same ratio for the whole sample helped to assess how each of the five forms distinguished itself from the average form. Five arch forms were distinguished as narrow, wide, mid, pointed and flat.

Table 3: Mean values for the five ratios used in the determination of arch form

\begin{tabular}{|c|c|c|c|c|}
\hline \multirow{2}{*}{ Ratio } & \multicolumn{3}{|c|}{ Maxillary } & \multicolumn{2}{c|}{ Mandibular } \\
\cline { 2 - 4 } & Female & Male & 0.19 & 0.21 \\
\hline$\frac{\text { Anterior arch length }}{\text { Inter canine distance }}$ & 0.26 & 0.26 & 0.58 \\
\hline$\frac{\text { Molar-vertical distance }}{\text { Inter-first molar distance }}$ & 0.60 & 0.57 & 0.76 \\
\hline$\frac{\text { Total arch length }}{\text { Inter-second molar distance }}$ & 0.77 & 0.73 & 0.71 \\
\hline$\frac{\text { Inter- canine distance }}{\text { Inter-first molar distance }}$ & 0.67 & 0.67 & 0.59 & 0.57 \\
\hline$\frac{\text { Inter-first molar distance }}{\text { Inter-second molar distance }}$ & 0.72 & 0.70 & 0.65 & 0.65 \\
\hline
\end{tabular}

The distribution of subjects according to five arch forms (Figure 2) is more or less balanced with a frequency of each group varying between $9.3 \%$ and $30.9 \%$ showing predominance of narrow arch form and fewer tendencies for mid arch form. The narrow form $(30.9 \%)$ is the most frequent arch form followed by wide arch form $(23.9 \%)$ whereas mid form $(9.3 \%)$ is the least frequent in both gender. Pointed and flat arch forms are relatively equally distributed. Narrow arch form is rather more common in females. The sex distribution was significantly different (Chi-square=16.218, d.f=4, p-0.002), Table 4.

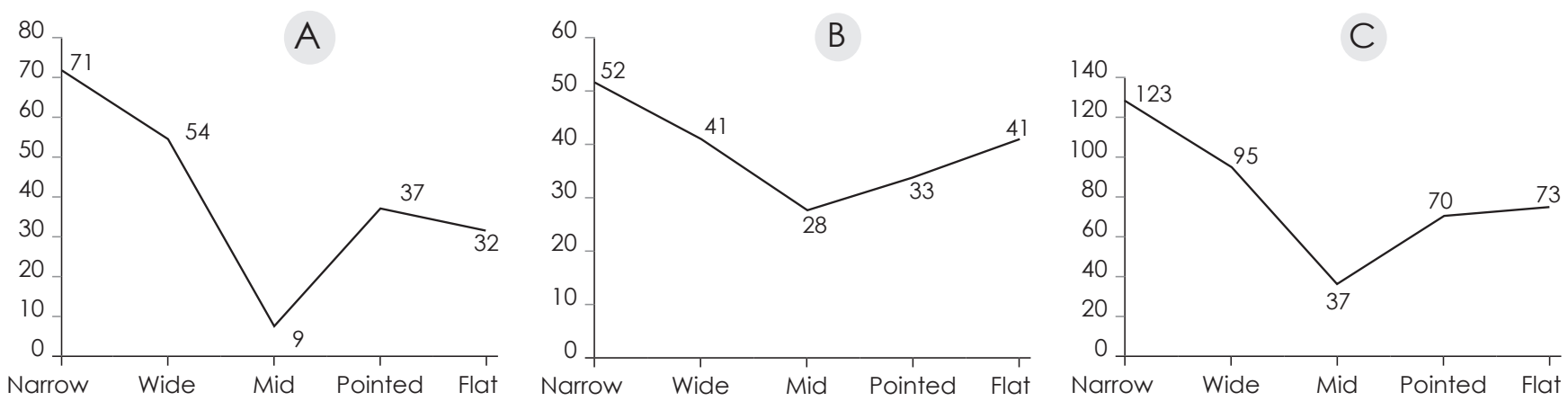

Figure 2: Distribution of the five dental arch forms. A: Female; B: Male; C: Total sample 
Table 4: The distribution of subjects according to arch forms

\begin{tabular}{|c|c|c|c|c|}
\hline \multirow{2}{*}{ Form } & Female & Male & Total & \multirow{2}{*}{$p$-value } \\
\hline & n (\%) & n (\%) & n (\%) & \\
\hline Narrow & 72 (17.4) & 51 (13.5) & $123(30.9)$ & \multirow{6}{*}{$0.002^{*}$} \\
\hline Wide & $54(13.6)$ & 41 (10.3) & 95 (23.9) & \\
\hline Mid & $9(2.3)$ & $28(7.0)$ & $37(9.3)$ & \\
\hline Pointed & $37(9.3)$ & 33 (8.3) & 70 (17.6) & \\
\hline Flat & $32(7.8)$ & 41 (10.5) & 73 (18.3) & \\
\hline Total & $204(50.4)$ & $194(49.6)$ & $398(100)$ & \\
\hline
\end{tabular}

*Significance level $p<0.05$.

\section{DISCUSSION}

Establishment of the most frequent dental arch form is useful in both diagnosis and construction or choosing the ready-made arch wires. "The inter-canine distance was selected because it was stable in the maxillary and mandibular arches after 13 years of age and the studies on retention and relapse stressed the need of stable unchangeable inter-canine width. ${ }^{13}$ At the same time the inter-canine width with the anterior arch length accurately describe the arch form anteriorly (pointed or flat).

The inter-first molar distance gives full picture about the widths of the arch, enabling to distinguish between narrow and wide arches especially when using the inter-canine distance/interfirst molar distance ratio. The inter-second molar distance was not given the same concern as the inter-first molar distance in previous studies as they assumed that the arch width distal to the first molar was similar to that of inter-first molar width and was not an area of importance as most of the orthodontic appliances deal with the arch wire mesial to the second molar without incorporating it in the treatment plan. However we should not forget that we are dealing with a full complement of permanent teeth. Also, many studies performed on commercially produced preformed wires concluded that almost all wires tend to narrow or being unchangeable distal to the first molar while the dental arch tend to widen posteriorly, so the second molar width was taken into consideration in the present study. It is worth mentioning that the greatest difference between male and female exists in the inter-second molar distance in both dental arches, which may be attributed to the difference in arch form. Thus to complete the skeleton of the arch form; three arch length dimensions were selected including: anterior, molar-vertical distance and total arch length.

In general, it was obvious that the mean values of all measurements taken for the dental arch dimensions confirmed the accepted view that the maxillary dental arch was larger in all dimensions than the mandibular counterpart. ${ }^{14-16}$ Since Angle; ${ }^{17}$ orthodontists have tried to determine a single ideal arch form that can ensure the stability of the therapeutic results. The findings of the present study confirm that the ideal dental arch form has not a single and universal form, but that there are at least five following different forms in untreated adults with normal occlusion.

Form (1); Narrow: Characterized by higher values of the sagittal/transverse ratios when compared with the mean values for these ratios, which indicates relative increase in arch lengths when compared with arch widths.

Form (2); Wide: Characterized by lower values of the sagittal/ transverse ratios when compared with the mean values for those ratios, which indicates relative increase in arch widths when compared with arch lengths.

Form (3); Mid: Characterized by non-significantly deviated values of the sagittal/transverse ratios when compared with the mean values.

Form (4); Pointed: Characterized by noticeably high values of anterior arch length/inter-canine distance ratio, which indicates relative increase of anterior arch length and relative decrease in the inter-canine distance.

Form (5) Flat: This form is opposite to the pointed form and characterized by a noticeably low value of anterior arch length/inter-canine distance ratio, which indicates relative decrease of anterior arch length and relative increase in the inter-canine distance.

According to Proffit ${ }^{15}$ dental arch form consists of dental units arranged in unique positions along a compound curve which represents a steady state of equilibrium delimited by the counter balancing force fields of the tongue and of the circum-oral tissues. Studying the descriptive analysis for the dental arch width revealed that the coefficient of variation (CV) values for all measurements are nearly close to each other, with the inter-canine distances showing the higher CV than the others. The inter-canine distance is responsible for the different arch forms.

It can be also noticed from the descriptive analysis of the dental arch length that the CV values for all measurements are nearly close to each other, with the anterior arch length 
showing the highest $C \vee$ value. The anterior arch length values share the inter-canine distance values through the ratio in the contribution of determining all types of the dental arch forms, and it is the most important ratio that determines the five dental arch forms. So, it is not unexpected for the anterior arch length to have the highest coefficient of variation among other dimensions. These results are in agreement with conclusions of Andria and Carlos. ${ }^{18}$

The predominance of the narrow type in both maxillary and mandibular dental arches, in the present study is not in agreement with the findings of Patel et $\mathrm{al}^{19}{ }^{19}$ who found that the most frequent maxillary form is the mid form and Burris and Harris ${ }^{20}$ who found that the most frequent mandibular arch form are squarer in American blacks in the canine-premolar region, while data collected by Felton and Sinclair $^{2}$ revealed the predominance of the narrowest arch form.

\section{CONCLUSION}

The arch form could possibly be clustered and used to describe each form of the maxillary and the mandibular arches. Five arch forms: narrow, wide, mid, pointed and flat were distinguished as unique forms for the maxillary and the mandibular arches. In the present sample of Yemeni adult the most prevalent form is the narrow form in both male and female samples.

\section{REFERENCES}

1. Musich DR, Ackerman JI. The catenometer: A reliable device for estimating dental arch perimeter. Am J Orthod 1973; 63:366-375.

2. Felton JM, Sinclair PM. A comutenized analysis of the shape and stability of mandibular arch form. Am J Orthod 1987; $92: 478-483$.

3. Brader AC. Dental arch form related with intraoral forces: PR=C. Am J Orthod 1972; 61:541-561.

4. Ferrario V, Sforza C, Miani A, Tartaglia G. Mathematical definition of the shape of dental arches in human permanent healthy dentitions. Eur J Orthod 1994; 16:287-294.

5. Currier JH. A computerized geometric analysis of human dental arch form. Am J Orthod 1969; 56:164-179.

6. White LW. Individualized ideal arches. J Clin Orthod 1978; 12:779-787.

7. Rudge SJ. A computer program for the analysis of study models. Euro J Orthod 1982; 4:269-273.

8. Hawley CA. Determination of the normal arch, and its application to orthodontia. Dent Cosmos 1905; $38: 541-552$.

9. Bonwill WG. Geometrical and mechanical laws of articulation. Transactions of the Odontological society of Pennsylvania 1885; $119: 133$.

10. Lavelle CL. A metrical study of dental arch form. J Dent 1978; 6:120-124.

11. Raberin M, Laumon B, Martin J, Brunner F. Dimensions and form of dental arches in subjects with normal occlusion. Am J Orthod Dentofacial Orthop1993; 103: 67-72.

12. Begole EA, Fox DL, Sadowsky C. Analysis of change in arch form with premolar expansion. Am J Orthod Dentofac. Orthop 1998; 113:307315.

13. Bishara SE, Jakobsen J R, Treder J, Nowak A. Arch width changes from 6 weeks to 45 years of age. Am J Orthod. Dentofac Orthop1997; 111:401-409.

14. Sillman JH. Dimensional changes of dental arches: Longitudinal study from birth to 25 years. Am J Orthod 1964; 50:824-842.

15. Proffit WR. Etiology of malocclusion, contemporary orthodontics (4th ed). St Louis: Mosby 2007; pl 45-49.

16. Eid A, El-Namrawy M, Kadry W. The relationship between the width, depth and circumference of the dental arch for a group of Egyptian school children. Egypt Orthod J 1987; 1:113-136.

17. Angle EH. Treatment of malocclusion of the teeth. 7th ed. Philadelphia, S.S. W. B. Saunders Company: 1907.

18. Andria LM, Carlos J. Relation of maxillary and mandibular intercuspid width, to bizygomatic and bigonial breadth. Angle Orthod1978; 48:154-162.

19. Patel VJ, Bhatia AF, Mahadevia SM, Italia S, Vaghamsi M. Dental Arch Form Analysis in Gujarati Males and Females having Normal Occlusion. J Ind Orthod Soc 2012; 46(4):295-9.

20. Burris B, Harris E. Maxillary arch size and shape in american blacks and whites. Angle Orthod 2000; 70:297-302. 